\title{
Microplasmas, a platform technology for a plethora of plasma applications
}

\author{
Kurt Becker ${ }^{\mathrm{a}}$ \\ Department of Applied Physics and Department of Mechanical and Aerospace Engineering, \\ New York University Tandon School of Engineering, Brooklyn, NY 11201, USA
}

Received 27 November 2016

Published online 3 August 2017

Publications describing microplasmas, which are commonly defined as plasmas with at least one dimension in the submillimeter range, began to appear to the scientific literature about 20 years ago. As discussed in a recent review by Schoenbach and Becker [1], interest and activities in basic microplasma research as well as in the use of microplasma for a variety of application has increased significatly over the past 20 years. The number of papers devoted to basic microplasma science increased by an order of magnitude between 1995 and 2015, a count that excludes publications dealing exclusively with technological applications of microplasmas, where the microplasma is used solely as a tool. In reference [1], the authors limited the topical coverage largely to the status of microplasma science and our understanding of the physics principles that enable microplasma operation and further stated that the rapid proliferation of microplasma applications made it impossible to cover both basic microplasma science and their application in a single review article.

Some microplasmas are thermal in nature, where the gas temperature is far above the room temperature and is approaching the electron temperature. Another group of microplasmas is nonthermal (also referred to as nonequilibrium or "cold"), with gas temperatures much below electron temperatures. Their electron energy distribution contains a tail of high energy electrons, which causes high excitation, ionization, and dissociation rates. Research into nonthermal microplasmas has enjoyed an enormous growth in the past two decades. These plasmas are particularly attractive for a plethora of applications because they can be operated stably at high gas pressures, in rare gases as well as in molecular gases and gas mixtures, operated in a direct current (dc) mode as well as in pulsed dc and alternate current (ac) modes. Modeling and improved diagnostics have allowed us in the past decade to gain more insight into the specific properties of nonthermal micro-plasmas. Electron densities exceeding $10^{16} \mathrm{~cm}^{-3}$ have been measured in pulsed microplasmas [1]. Gas temperatures, on the other hand, can be close to room temperature at low currents in rare gases and generally reach not more than $2000 \mathrm{~K}$ in atmospheric-pressure air microplasmas. With discharge voltages of a few hundred volts, and current densities on the order of

${ }^{a}$ e-mail: kurt.becker@nyu.edu 
$10 \mathrm{~A} / \mathrm{cm}^{2}$ (at currents of $1 \mathrm{~mA}$ in a microplasma device with a $100 \mu \mathrm{m}$ cathode opening), the power densities in such a microplasma with typical dimensions of $100 \mu \mathrm{m}$ can reach values on the order of $10^{5} \mathrm{Wcm}^{-1}$ [1]. Progress into the basic science as well as some applications in the first 10 years was summarized in a few reviews [2-7]. The latter review [7] also discussed transient, filamentary plasmas.

The nonthermal nature of microplasmas in conjunction with the possibility of stable high-pressure operation, high power density, non-Maxwellian electron energy distributions with a significant component of energetic electrons, low gas temperatures (down to ambient temperature), and the possibility to build two-dimensional arrays without individual ballasting have made it possible to create microplasma devices for a wide range of applications. A number of reviews focused on selected areas of microplasma applications [8-13]. The development of excimer lamps with emissions in the VUV was one of the first application-oriented developments using microplasmas [7]. The same basic mechanism, three-body reactions, forms the basis for microplasmabased ozone generators [14].

The resistive character of microplasmas under certain operating conditions giving rise to the creation of large microplasma-arrays made possible flat panel light sources with more than $10^{5}$ individual microplasmas. Addressable flat panels have also been developed based on the same fabrication method. An overview of recent developments of these devices can be found in reference [9]. Another area where microplasmas have been successfully introduced is in material processing, particularly the synthesis of nanoparticles $[15,16]$. Plasma medicine is an emerging area of application, which has gained strongly in interest by the scientific community. Reviews on this topic have been published as early as 2005, and continue to appear regularly in the literature. However, many of these reviews typically cover only parts of this expanding research area [17-20]. Major advances were made possible by the development of plasma sources that are biocompatible (i.e., satisfy strict conditions in terms of their electrical, chemical, and thermal properties), consist of biocompatible materials, and can generate copious amounts of reactive radicals and deliver them to biological tissue via plasma jets. We note that medical applications of plasmas also bear risks, which should be properly assessed [21]. Besides medical applications, studies on environmental applications have been performed (see e.g., Becker [22] and references therein). Lesser known, but important applications of microplasmas, are in the area of detectors [8], and even microthrusters [23].

It should perhaps be noted that the rapid development of microplasma applications has often occurred by trial-and-error without having a good understanding of the underlying basic science. Plasma applications as diverse as surface modification and functionalization, light sources, ozone generation, pollution control, biological inactivation, sensors rely on the plasma-initiated generation of chemically reactive species, in particular reactive oxygen and nitrogen species. We only have an understanding of some of the key plasma chemical reaction pathways that drive the process in a few applications. Moreover, in almost all areas of application we lack a detailed knowledge of the coupling of the plasma physics to the plasma chemistry. We do not yet have the level of understanding of the basic plasma physics that will allow us to tailor the plasma parameters such as plasma composition and gas temperature, power density, electron density, and electron energy distribution to selectively maximize the generation of certain plasma species, while minimizing or suppressing the formation of others. Thus, we lack the ability to achieve control of the plasma chemistry in many applications by making use of our insight into the basic plasma physics.

That said, applications can also be powerful drivers and motivators for basic science and the development of new plasma sources. As an example, demands for plasma 
sources with very application-specific operating parameters and performance characteristics have led to the development of a wide range of microplasma sources [1] starting with the original concepts of a microplasma generated between parallel-plate electrodes that included dc microplasmas between a flat cathode and a ring-shaped electrode (so-called cathode boundary layer plasmas), and ac (microwave) microdischarges between parallel edge electrodes. Very quickly, the need arose for generating and sustaining microplasmas in two-dimensional microcavity electrode structures excited by dc, ac, and pulsed power. By now, various microplasma arrays have been demonstrated with arrangements where the individual microplasmas are operated in series or in parallel. Another variant of microplasma sources based on the so-called "capillary plasma electrode" concept was developed specifically for chemical processing, decontamination, and medical sterilization applications $[1,24]$.

The need for plasma treatment of objects, whose size and shape did not allow them to be placed in the microplasma gaps for direct place treatment but rather required remote plasma treatment, led to the advent of various types of atmosphericpressure plasma jets (APPJs). APPJs have gained wide spread use in the past 10 years. Summaries of the performance characteristics of the most commonly employed APPJs can be found in several excellent reviews [25-27]. In the rapidly developing field of plasma medicine, the excitement stemming from successful applications such as seeing how the use of a microplasma can accelerate blood coagulation or stimulate wound healing has triggered many studies into identifying the underlying chemical and biological reaction path ways initiated by the plasma. Developments in the field of (bio)medical applications of plasmas have given rise to new journals, such as "Plasma Medicine", which started in 2011 and is devoted entirely to papers on plasma medicine [28] and "Clinical Plasma Medicine" [29].

This issue contains articles representing various areas of microplasma-enabled applications ranging from biological and biomedical applications including bacterial inactivation to new plasma-based devices such as ion sources, hall thrusters, and two-dimensional plasma jet arrays to ozone generation. Martus and co-workers [30] used an atmospheric-pressure He plasma to study the regeneration of an amputated tadpole tail with an emphasis of elucidating the role of reactive oxygen species, antioxidant defenses, and morphological features. The plasma treated tail showed an increased growth rate, elevated reactive oxygen species and increased antioxidant defenses in the regenerate as opposed to the untreated control. Szili et al. [31] report a new approach to control protein and cell attachment inside 96 -well polystyrene plates. Bovine serum albumin (BSA) coated wells were treated with a He microplasma jet for different times to remove the BSA from the surface in a controlled fashion. This, in turn, provided control of the level of enzyme and cell attachment and induced a new surface chemistry. These results open new avenues for high-throughput screening of new cell culture platforms where control over the level of protein, enzyme or cell adherence is needed in order to maintain a specific cell function. Fang and collaborators [32] studied the synergistic effect of plasma activated water (PAW) and hydrogen peroxide $\left(\mathrm{H}_{2} \mathrm{O}_{2}\right)$, PAW/HP, in the sterilization of $\mathrm{S}$. aureus bacteria. They observed that $\mathrm{PAW} / \mathrm{HP}$ as compared to PAW shows significantly higher concentrations of reactive oxygen and nitrogen species, which, in turn, results in a significantly higher inactivation efficacy. Zhang and co-workers [33] showed that a low-temperature microplasma can serve to disinfect cardiac implantable pace makers with high efficacy.

Brandenburg and Saranani [34] studied details of the conversion of the greenhouse gas carbon dioxide to carbon monoxide in a Dielectric Barrier Discharge (DBD) in $\mathrm{CO}_{2}$ and $\mathrm{CO}_{2} / \mathrm{N}_{2}$ gas mixtures. These authors analyzed a single filament DBD arrangement operated under sinusoidal high-voltage by means of spectroscopic and electrical diagnostics with high spatial and temporal resolution and sensitivity. They 
found that the filament development is similar in air or other nitrogen-oxygen gas mixtures, although the gas composition influences the duration and other filament parameters. On the other hand, increasing the $\mathrm{CO}_{2}$ content in the gas mixture leads to weaker filaments and faster quenching of excited molecular states. The optimum power dissipation into single discharge is obtained for a $\mathrm{CO}_{2}$ content between 20 and $30 \%$ by volume. Eden and co-workers [35] presented the results of ozone $\left(\mathrm{O}_{3}\right)$ generation in a new embodiment consisting of an array of thousands of microchannel plasmas simultaneously, in a so-called plasma chip. This device consists of a flat aluminum strip fabricated by photolithographic and wet chemical processes and comprises 24-48 channels micromachined into nanoporous aluminum oxide, and embedded electrodes. The authors showed that the combined output of 18 modules comprising 72 chips and 1,800 microchannels produced $115 \mathrm{~g} / \mathrm{hr}(2.7 \mathrm{~kg} /$ day $) \mathrm{O}_{3}$. Efficiencies exceeding $130 \mathrm{~g} / \mathrm{kWh}$ when oxygen is used the feedstock gas can be achieved with a high degree of stability and reliability.

Ding et al. [36] reported the properties of a 200 Watt, no-Wall-Losses Hall Thruster based on a novel design that verifies the positive effect of "pushing down" the magnetic field with two permanent magnetic rings. These authors investigated the thrust, discharge current, specific impulse, and efficiency and showed that "no erosive discharge" of the wall is achieved in the range of $120-460 \mathrm{~W}$ with a maximum efficiency of the anode of about 50\%. Xia et al. [37] describe the properties of an ion source based on a wire-to-plate corona discharge plasma for an Ion Mobility Spectrometer (IMS). They studied the characteristics of the corona discharge and the ion current detected on a Faraday plate for different electrode spacings and voltages. The present IMS provides a much larger ion current compared to a point-to-plate corona design.

Zhu and co-workers [38] showed that an AC atmospheric-pressure plasma jet generated in noble gas and noble gas $/ \mathrm{O}_{2}$ admixture as working gas has properties similar to those of DC-excited plasma bullets. Bullet speeds in access of $100 \mathrm{~m} / \mathrm{s}$ were achieved. Multiple radial jets could be realized with dielectric tubes of different sizes. As a case study, one of these multi-jet devices was used to treat B. aureus on the inner surface of a plastic beaker and was shown to be much more effective than a single jet. $\mathrm{Lu}$ et al. [39] used non-invasive Stark polarization spectroscopy of the He $447.1 \mathrm{~nm}$ line to determine the electric field in an atmospheric-pressure microplasma jet driven by positive pulsed DC power with a pulse rising time of $60 \mathrm{~ns}$. They determined the electric field strength in the streamer head $\left(\mathrm{E}_{h}\right)$ to be in the range of $9-17 \mathrm{kV} / \mathrm{cm}$, which was found to first increase as the streamer head emerges from the tube exit and then decreases after reaching a maximum of $17 \mathrm{kV} / \mathrm{cm}$. A similar tendency was observed for the streamer velocity.

This issue gives but a small glimpse into the diverse range of applications that were made possible by the advent of microplasmas. The development of these applications up to now and the potential for their future advancement have greatly benefitted from the insights obtained from concurrent research activities devoted to basic microplasma science. Likewise, new results from the application of microplasmas, often obtained without clear understanding of the underlying plasma science principles, and new fields of use for microplasmas yet to developed, will stimulate additional basic microplasma research. Thus, further developments in micro-plasma science and the use of microplasmas in applications can be expected to go hand-in-hand and enjoy a mutually beneficial, symbiotic relationship.

I would like to thank my co-guest editors of this issue, Dr. Ronny Brandenburg, Prof. J. Gary Eden, Dr. XinPei Liu, and Prof. WeiDong Zhu for their dedication and efforts to help make this issue of EPJ Special Topics a success. 


\section{References}

1. K.H. Schoenbach, K. Becker, Eur. Phys. J. D 70, 29 (2016)

2. R. Foest, M. Schmidt, K. Becker, Microplasmas Int. J. Mass Spectrom. 248, $87(2005)$

3. K.H. Becker, K.H. Schoenbach, J.G. Eden, J. Phys. D: Appl. Phys. 39, R55 (2006)

4. K. Tachibana, IEEJ Trans. 1, 145 (2006)

5. F. Iza, G.J. Kim, S.M. Lee, J.K. Lee, J.L. Walsh, Y.T. Zhang, M.G. Kong, Plasma Proc. Polymers 5, 322 (2008)

6. A.P. Papadakis, S. Rossides, A.C. Metaxas, The Open Appl. Phys. J. 4, 45 (2011)

7. U. Kogelschatz, J. Opt. Technol. 79, 484 (2012)

8. K.H. Schoenbach, W. Zhu, IEEE J. Quantum Electr. 48, 768 (2012)

9. C.K. Eun, Y. Gianchandani, IEEE J. Quantum Electr. 48, 814 (2012)

10. J.G. Eden, S.-J. Park, J.H. Cho, M.H. Kim, T.J. Houlahan, Jr., B. Li, E.S. Kim, T.L. Kim, S.K. Lee, K.S. Kim, J.K. Yoon, S.H. Sung, P. Sun, C.M. Herring, C.J. Wagner, IEEE Trans. Plasma Sci. 41, 661 (2013)

11. X. Lu, M. Laroussi, V. Puech, Plasma Sources Sci. Techn. 21, 034005 (2012)

12. X. Lu, G.V. Naidis, M. Laroussi, K. Ostrikov, Phys. Rep. 540, 123 (2014)

13. J. Hopwood, A.R. Hoskinson, J. Gregorio, Plasma Sources Sci. Technol. 23, 064002 (2014)

14. K.S. Kim, S.-J. Park, J.G. Eden, J. Phys. D 41, 012004 (2008)

15. D. Mariotti, R.M. Sankaran, J. Phys. D: Appl. Phys. 43, 23001 (2010)

16. R. Wang, S. Zuo, D. Wu, J. Zhang, W. Zhu, K. Becker, J. Fang, Plasma Proc. Poly. 12, $380(2015)$

17. K. Becker, A. Koutsospyros, S.-M. Yin, C. Christodoulatos, N. Abramzon, J.C. Joaquin, G. Brelles-Mariño, Plasma Phys. Controlled Fusion 47, B513 (2005)

18. M.G. Kong, G. Kroesen, G. Morfill, T. Nosenko, T. Shimizu, J. van Dijk, J.L. Zimmermann, New J. Phys. 11, 115012 (2009)

19. M. Laroussi, IEEE Trans. Plasma Sci. 43, 703712 (2015)

20. T. von Woedtke, S. Reuter, K. Masur, K.D. Weltmann, Phys. Rep. 530, 291 (2013)

21. J. Lademann. C. Ulrich, A. Patzelt, H. Richter, F. Kluschke, M. Klebes, O. Lademan, A. Kramer, K.D. Weltman, B. Lange-Asschenfeldt, Clinical Plasma Medicine 1, 5 (2013)

22. K. Becker, The Use of Nonthermal Plasmas in Environmental Applications, chapter 15 in Introduction to Complex Plasmas, Vol. 59 in Series on Atomic, Optical, and Plasma Physics, edited by M. Bonitz, N. Horing, and P. Ludwig (Springer-Verlag, Heidelberg, 2010)

23. M.J. Kushner, J. Phys. D: Appl. Phys. 38, 1633 (2005)

24. E.E. Kunhardt, K. Becker, US Patents 5872426, 6005349, and 6147452 (1999)

25. M. Laroussi, T. Arkan, Plasma Proc. Polym. 4, 777 (2007)

26. K.D. Weltmann, E. Kindel, T. von Woedtke, M. Hähnel, M. Stieber, R. Brandenburg, Pure Appl. Chem. 82, 1223 (2010)

27. G.Y. Park, S.J. Park, M.Y. Choi, I.G. Koo, J.H. Byun, J.W. Hong, J.Y. Sim, G.J. Collins, J.K. Lee, Plasma Sources Sci. Techn. 21, 043001 (2012)

28. Plasma Medicine, edited by Gregory Fridman (Begell House Publications, 2011)

29. Clinical Plasma Medicine, edited by Hans-Robert Metelmann and Thomas v. Woedtke (Elsevier, 2013)

30. A. Rivie, K. Martus, J. Menon, Eur. Phys. J. Special Topics 226, 2859 (2017)

31. E.J. Szili, S. Becker, R.D. Short, S.A. Al-Bataineh, Eur. Phys. J. Special Topics 226, $2873(2017)$

32. S. Wu, Q. Zhang, R. Ma, S. Yu, K. Wang, J. Zhang, J. Fang, Eur. Phys. J. Special Topics 226, 2887 (2017)

33. Y. Zhang, Y. Li, Y. Li, S. Yu, H. Li, J. Zhang, Eur. Phys. J. Special Topics 226, 2901 (2017)

34. R. Brandenburg, A. Sarani, Eur. Phys. J. Special Topics 226, 2911 (2017) 
35. M.-H. Kim, J H. Cho, S.-J. Park, J.G. Eden, Eur. Phys. J. Special Topics 226, 2923 (2017)

36. Y. Ding, W. Peng, H. Sun, L. Wei, M. Zeng, F. Wang, D. Yu, Eur. Phys. J. Special Topics 226, 2945 (2017)

37. Q. Xia, Y. Zhang, J. Ouyang, Eur. Phys. J. Special Topics 226, 2955 (2017)

38. W. Zhu, R. Wang, Eur. Phys. J. Special Topics 226, 2965 (2017)

39. Y. Lu, S. Wu, W. Cheng, X. Lu, Eur. Phys. J. Special Topics 226, 2979 (2017) 